\title{
Erythropoietin ameliorates hyperglycemia in type I-like diabetic rats
}

This article was published in the following Dove Press journal:

Drug Design, Development and Therapy

3 June 2016

Number of times this article has been viewed

\author{
Ho-Shan Niu' \\ Chin-Hong Chang ${ }^{2,3}$ \\ Chiang-Shan Niu' \\ Juei-Tang Cheng $g^{3,4}$ \\ Kung-Shing Lee L-7 $^{5-4}$
}

'Department of Nursing, Tzu Chi University of Science and Technology, Hualien City, Taiwan, Republic of China; ${ }^{2}$ Department of Neurosurgery, ${ }^{3}$ Department of Medical Research, Chi-Mei Medical Center, Yong Kang, Tainan City, Taiwan, Republic of China; ${ }^{4}$ Institute of Medical Sciences, Chang Jung Christian University, Gueiren, Tainan City, Taiwan, Republic of China; ${ }^{5}$ Department of Surgery, Division of Neurosurgery, Pingtung Hospital, Pingtung, Taiwan, Republic of China; ${ }^{6}$ Department of Surgery, Kaohsiung Medical University Chung-Ho Memorial Hospital, Kaohsiung City, Taiwan, Republic of China; ${ }^{7} \mathrm{~S} c h o o l$ of Medicine, Kaohsiung Medical University, Kaohsiung City, Taiwan, Republic of China

\begin{abstract}
Background: Erythropoietin (EPO) is widely used in diabetic patients receiving hemodialysis. The role of EPO in glucose homeostasis remains unclear. Therefore, we investigated the effect of EPO on hyperglycemia in rats with type 1-like diabetes.

Methods: Rats with streptozotocin-induced type 1-like diabetes (STZ rats) were used to estimate the blood glucose-lowering effects of EPO, and changes in the expression levels of glucose transporter 4 (GLUT4) and the hepatic enzyme phosphoenolpyruvate carboxykinase (PEPCK) were identified by Western blot analysis.
\end{abstract}

Results: EPO attenuated the hyperglycemia in the STZ rats in a dose-dependent manner without altering the hematopoietic parameters, including the hematocrit and number of red blood cells. The involvement of the EPO receptor (EPOR) was identified using EPOR-specific antibodies. In addition, injection of EPO enhanced the glucose utilization, which was assessed using an intravenous glucose tolerance test in rats. However, blood insulin was not changed by EPO in this assay, showing the insulinotropic action of EPO. Moreover, EPO treatment increased the insulin sensitivity. Western blots indicated that the phosphorylation of AMP-activated protein kinase was enhanced by EPO to support the signaling caused by EPOR activation. Furthermore, the decrease in the GLUT4 level in skeletal muscle was reversed by EPO, and the increase in the PEPCK expression in liver was reduced by EPO, as shown in STZ rats.

Conclusion: Taken together, the results show that EPO injection may reduce hyperglycemia in diabetic rats through activation of EPO receptors. Therefore, EPO is useful for managing diabetic disorders, particularly hyperglycemia-associated changes. In addition, EPO receptor will be a good target for the development of antihyperglycemic agent(s) in the future.

Keywords: erythropoietin, GLUT4, PEPCK, STZ rats

\section{Introduction}

Erythropoietin (EPO), a $30.4 \mathrm{kDa}$ growth factor, is mainly produced in the kidney and stimulates erythropoiesis in bone marrow. ${ }^{1}$ Recombinant human EPO is an effective treatment for anemia of various origins, including anemia associated with renal failure $^{2}$ and cancer-related diseases. ${ }^{3}$ The major function of EPO is mediated by a specific cell-surface receptor, EPO receptor (EPOR). In multiple tissues, the expression of EPORs has been correlated with the effectiveness of EPO in nonhematopoietic tissues, including the brain ${ }^{4}$ and peripheral tissues ${ }^{5,6}$ Furthermore, in the heart, EPO protects cardiomyocytes against ischemic injury, ${ }^{7}$ and this nonhematopoietic effect is described as a pleiotropic action of EPO. ${ }^{8}$

In clinics, EPO is widely used in hemodialysis for patients with nephropathy, mainly due to diabetes, and this application established the need for critical exploration of the interplay between EPO and glucose in the absence of clinical problems. ${ }^{9}$ Hyperglycemia is a central factor in the induction of diabetic disorders, including nephropathy. ${ }^{10}$ 
Earlier glycemic control reduced the incidence of diabetic nephropathy, ${ }^{11}$ and hyperglycemic damage to mesangial cells is implicated in the development of diabetic nephropathy. ${ }^{12}$ EPO is widely used in diabetic patients with chronic kidney disease. ${ }^{13}$ The positive effect of EPO on glucose homeostasis was reported during the hemodialysis in clinics. ${ }^{8}$ In addition, the effects of EPO on lipid metabolism ${ }^{14}$ and glucose intolerance ${ }^{15}$ were also observed. Therefore, the effect of EPO on diabetes has been researched, and the results were summarized in a recent review article. ${ }^{16}$

However, fewer studies have been conducted on EPOinduced reductions in hyperglycemia, except one study demonstrating the effects in mice. ${ }^{17}$ Therefore, in the present study, we investigated the effect of EPO on hyperglycemia using type 1-like diabetic rats with severely diminished circulating insulin levels. ${ }^{18}$

\section{Materials and methods Experimental animals}

Male Wistar rats weighing 260-280 g were obtained from the Animal Center of National Cheng Kung University Medical College. All rats were housed individually in plastic cages under standard laboratory conditions. The rats were maintained under a 12-hour light/dark cycle and had free access to food and water. All experiments were performed under anesthesia with sodium pentobarbital $(35 \mathrm{mg} / \mathrm{kg}$, intraperitoneal [ip]), and all efforts were made to minimize the animals' suffering. The animal experiments were approved and conducted in accordance with local institutional guidelines for the care and use of laboratory animals at Chi-Mei Medical Center. The experiments conformed to the Guide for the Care and Use of Laboratory Animals as well as the guidelines of the Animal Welfare Act.

\section{Induction of animal model}

As described in our previous report, ${ }^{19}$ overnight fasted rats were intravenously (iv) injected with streptozotocin (STZ; $60 \mathrm{mg} / \mathrm{kg}$ ) dissolved in $0.1 \mathrm{mmol} / \mathrm{L}$ citrate buffer ( $\mathrm{pH} 4.5$ ). One week later, blood samples from each rat were used to determine the glucose and insulin levels. Hyperglycemia and hypoinsulinemia were used to identify the success of this model, as described previously, ${ }^{19}$ and no mortality was observed during this induction.

\section{Drug treatment}

The stock solution of EPO containing epoetin beta (Recormon, 5,000 IU/0.3 mL) purchased from Roche (Mannheim, Germany) was diluted in 9\% normal saline. Antibodies for EPORs (Santa Cruz, Heidelberg, Germany) were used to block the EPORs. A fresh solution diluted to the indicated dose was applied to treat the animals. To rule out pharmacokinetic factors, EPO was iv injected at the indicated dose into anesthetized animals. The changes were then used for comparison with the control that was treated with the same volume of vehicle.

\section{Laboratory measurements}

The plasma glucose level was measured as described in our previous study. ${ }^{19}$ Blood samples $(0.2 \mathrm{~mL})$ were collected from the femoral vein of rats under anesthesia with sodium pentobarbital (35 mg/kg, ip). The blood samples were then centrifuged at 13,000 rpm for 3 minutes, and an aliquot $(15 \mu \mathrm{L})$ of plasma was added to $1.5 \mathrm{~mL}$ of glucose kit reagent (Biosystems SA, Barcelona, Spain) and incubated at $37^{\circ} \mathrm{C}$ for 10 minutes. The concentration of plasma glucose was measured in an analyzer (Quik-Lab, Ames; Miles Inc, Elkhart, IN, USA). The plasma insulin level was measured using a commercially available enzyme-linked immunosorbent assay kit (Linco, St Charles, MO, USA).

\section{Intravenous glucose tolerance test}

The intravenous glucose tolerance test (IVGTT) was performed according to our previous method with some modifications. ${ }^{20}$ Briefly, the rats fasted for 8 hours, and the basal plasma glucose concentration was obtained from samples from the femoral vein of rats under anesthesia. A saline EPO solution at the indicated dose or the same volume of saline was injected into the femoral vein of rats. Thirty minutes later, blood samples $(0.1 \mathrm{~mL})$ from the femoral vein were drawn, and this time point was indicated as 0 minute. Next, $0.5 \mathrm{~g}$ of glucose dissolved in $1 \mathrm{~mL}$ of saline was iv administered to rats at $0.5 \mathrm{~g} / \mathrm{kg}$. In another group, which served as the control, rats receiving a similar injection of saline at the same volume were used. Blood samples $(0.1 \mathrm{~mL})$ from the femoral vein were drawn 5, 10, 20, 30, 60, 90, and 120 minutes after the oral glucose loading for the plasma glucose measurement. The rats were maintained under anesthesia by pentobarbital (30 mg/kg, ip) throughout the experiments. Changes in the reduction of blood glucose (area under the curve [AUC]) were compared to determine the effect of EPO.

\section{Analysis of insulin sensitivity}

After fasting overnight, all rats were challenged with exogenous insulin. Similar to our previous method, ${ }^{21}$ the insulin challenge test was performed by an iv injection of short-acting human insulin at the indicated dose in rats. Blood samples $(0.2 \mathrm{~mL})$ from the femoral vein were drawn following the iv insulin challenge to measure the plasma glucose concentrations. 


\section{Western blotting analysis}

The expression of metabolic regulatory signals in skeletal muscle or liver was examined using Western blot analysis as described in our previous report. ${ }^{19}$ In brief, ice-cold radioimmunoprecipitation assay buffer containing phosphatase and protease inhibitors $(50 \mathrm{mM}$ sodium vanadate, $0.5 \mathrm{mM}$ phenyl methyl sulfonyl fluoride, $2 \mathrm{mg} / \mathrm{mL}$ aprotinin, and $0.5 \mathrm{mg} / \mathrm{mL}$ leupeptin) was used for protein extraction. The protein concentrations were then measured using a bicinchoninic acid protein assay kit (Thermo Fisher Scientific Inc, Waltham, MA, USA). The total protein samples ( $30 \mu \mathrm{g})$ were subjected to sodium dodecyl sulfate-polyacrylamide gel electrophoresis (10\% acrylamide gel) using the Bio-Rad Trans-Blot system (Bio-Rad, Hercules, CA, USA) and then transferred to membranes. The membranes were blocked with $5 \%$ nonfat milk in Tris-buffered saline containing $0.1 \%$ Tween 20 (TBS-T), incubated for 60 minutes, and then hybridized with primary antibodies specific to phosphoenolpyruvate carboxykinase (PEPCK) (Santa Cruz Biotech, Dallas, TX, USA) or glucose transporter 4 (GLUT4; Abcam, Cambridge, UK) and phospho-AMP-activated protein kinase (AMPK) or AMPK (Cell Signaling Technology, Beverly, MA, USA) at suitable concentrations in TBS-T for 16 hours. The membranes were then incubated with secondary antibody for an additional 3 hours. Incubation with secondary antibodies and detection of the antigen-antibody complex were performed using an enhanced chemiluminescence kit (Amersham Biosciences, Little Chalfont, UK), and $\beta$-actin (Merck Millipore, Darmstadt, Germany) was used as the internal control. After comparison with the marker to confirm specificity, the immunoblots of each spot were quantified with a laser densitometer.

\section{Statistical analysis}

Data are expressed as the mean \pm standard error of the mean. Statistical analysis was performed using one-way analysis of variance and Newman-Keuls post hoc analysis. $P$-values of 0.05 or less were considered significant.

\section{Results}

\section{Reduction of hyperglycemia by EPO in type I-like diabetic rats}

To understand the direct effect of EPO on glucose homeostasis, we employed rats with STZ-induced type 1-like diabetes as described in our previous report. ${ }^{19}$ Two weeks after the STZ injection, the rats became type 1-like diabetic, showing plasma glucose levels equal to or greater than $300 \mathrm{mg} / \mathrm{dL}$. In addition, the plasma insulin level in
STZ-induced diabetic rats was only $4 \%$ less than that in normal rats. Therefore, mediation by endogenous insulin is negligible in this type 1-like diabetic model, as described previously. ${ }^{19}$

Iv injection of EPO induced a marked reduction of hyperglycemia 30 minutes later, and this decrease was enhanced with time; it reached a plateau from 60 to 90 minutes later. At 120 minutes postinjection, this action of EPO gradually reversed, except at the highest dose. As shown in Figure 1, 60 minutes after the injection, EPO attenuated the hyperglycemia in the diabetic rats lacking insulin in a dose-dependent manner. After washout of the EPO, the hyperglycemia returned in these rats, and no irreversible action was observed. In addition, the hematocrit $(43.53 \% \pm 4.63 \%$ vs $43.66 \% \pm 2.06 \%$, $\mathrm{N}=8)$ and the number of red blood cells $\left(6.79 \pm 0.28 \times 10^{6} / \mu \mathrm{L}\right.$ vs $6.99 \pm 0.46 \times 10^{6} / \mu \mathrm{L}, \mathrm{N}=8$ ) were not modified by EPO at the highest dose in the treated diabetic rats.

\section{Blockade of EPO-induced hypoglycemia by antibodies specific to EPO receptors} in type I-like diabetic rats

A specific pharmacological antagonist for the EPO receptor has still not been developed. Therefore, as an alternative method, we employed antibodies specific to the EPOR to block the EPOR sites. As shown in Figure 2, the action of EPO was markedly inhibited by the pretreatment with antibodies specific to EPORs in diabetic rats, indicating that receptor activation mediates the EPO-induced actions.

\section{Effect of EPO on IVGTT in Wistar rats}

To evaluate the direct effect of EPO on glucose utilization, we performed an IVGTT as described previously. ${ }^{20}$ Thirty minutes after injection of EPO at the indicated dose, the plasma glucose values in blood samples were measured as

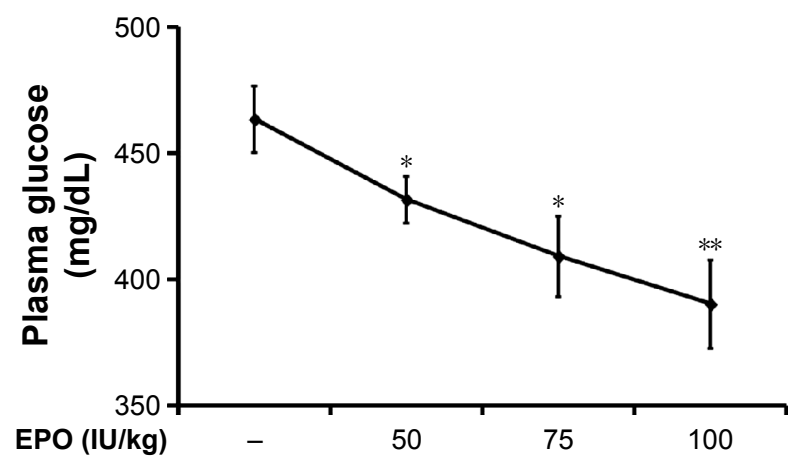

Figure I Effects of EPO on plasma glucose levels in type I-like diabetic rats. Notes: Dose-dependent changes in plasma glucose induced by EPO in rats with streptozotocin-induced diabetes are shown. Values (mean $\pm \mathrm{SE}$ ) were obtained from groups of eight animals. $* P<0.05$ and $* * P<0.01$ compared with the vehicle-treated diabetic rats.

Abbreviations: EPO, erythropoietin; SE, standard error. 


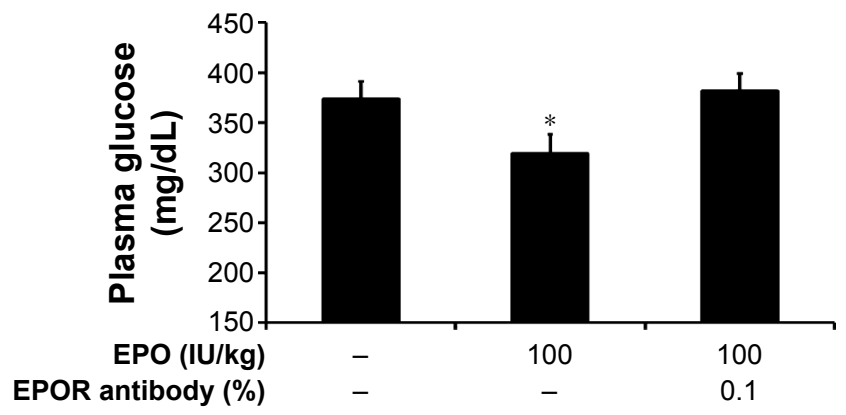

Figure 2 Blockade of EPO-induced actions by antibodies specific to EPORs in type I-like diabetic rats.

Notes: EPO-induced reduction of plasma glucose (middle column) reversed by antibodies specific to EPORs (right column) in rats with streptozotocin-induced diabetes. Values (mean $\pm \mathrm{SE}$ ) were obtained from groups of eight animals. ${ }^{* P}<0.05$ compared with the vehicle-treated diabetic rats.

Abbreviations: EPO, erythropoietin; EPOR, erythropoietin receptor; SE, standard error.

the 0 -minute time point. Then, $0.5 \mathrm{~g}$ of glucose dissolved in $1 \mathrm{~mL}$ of saline was iv injected into the rats at $0.5 \mathrm{~g} / \mathrm{kg}$. Compared with the vehicle-treated group, the EPO-treated group had a significantly lower plasma glucose concentration, particularly at 60 and 120 minutes (Figure 3A). The AUC for the change in plasma glucose was calculated, as shown in Figure 3B, and EPO was found to cause a dose-dependent reduction in plasma glucose. Essentially, hyperglycemia may induce insulin secretion. Then, the plasma insulin level was associated with blood glucose changes. Because the peak action of EPO was observed at 60 minutes in the IVGTT, we also determined the insulin level at the same time point and found that the plasma insulin was markedly $(P<0.05)$ increased from $1.42 \pm 0.10 \mu \mathrm{g} / \mathrm{L}(\mathrm{N}=8)$ to $2.96 \pm 0.07 \mu \mathrm{g} / \mathrm{L}$ $(\mathrm{N}=8)$. However, there was no difference in plasma insulin between the EPO-treated group $(2.89 \pm 0.09 \mu \mathrm{g} / \mathrm{L} ; \mathrm{N}=8)$ and the vehicle-treated group $(2.96 \pm 0.07 \mu \mathrm{g} / \mathrm{L})$, which suggested that the effect of EPO on IVGTT is not mediated by endogenous insulin in rats.

\section{EPO-induced enhancement of insulin sensitivity}

To understand the effect of EPO on insulin sensitivity, we investigated the responses to exogenous insulin, as previously described. ${ }^{5}$ As shown in Figure 4, the dosedependent hypoglycemia induced by exogenous insulin was also enhanced by EPO in diabetic rats. In particular, the response to higher doses of insulin was more markedly increased by EPO.

\section{Effect of EPO on GLUT4 changes in the skeletal muscle of STZ-diabetic rats}

Through our previously described method, ${ }^{19}$ the protein levels of GLUT4 in the skeletal muscle of these diabetic rats were determined. STZ-diabetic rats were treated with EPO $(0.5 \mathrm{mg} / \mathrm{kg} / \mathrm{d})$ for 1 week. Then, the STZ-diabetic rats were sacrificed by decapitation under anesthesia, and the soleus muscle was immediately isolated for Western blots. Similar to our previous report, ${ }^{20}$ the GLUT4 protein level in the soleus muscle was markedly reduced in STZ-diabetic rats (Figure 5A). Treatment of STZ-diabetic rats with EPO $(0.5 \mathrm{mg} / \mathrm{kg} / \mathrm{d})$ for 1 week resulted in an elevation of the GLUT4 protein level in the soleus muscle (Figure 5A). Therefore, EPO was found to demonstrate an elevation of GLUT4 expression in the skeletal muscle.

AMPK mediates the signaling of EPORs. ${ }^{16}$ Therefore, we also investigated the changes in phosphorylated AMPK (p-AMPK) in the skeletal muscle. As shown in Figure 5B, p-AMPK was markedly reduced in the soleus muscle
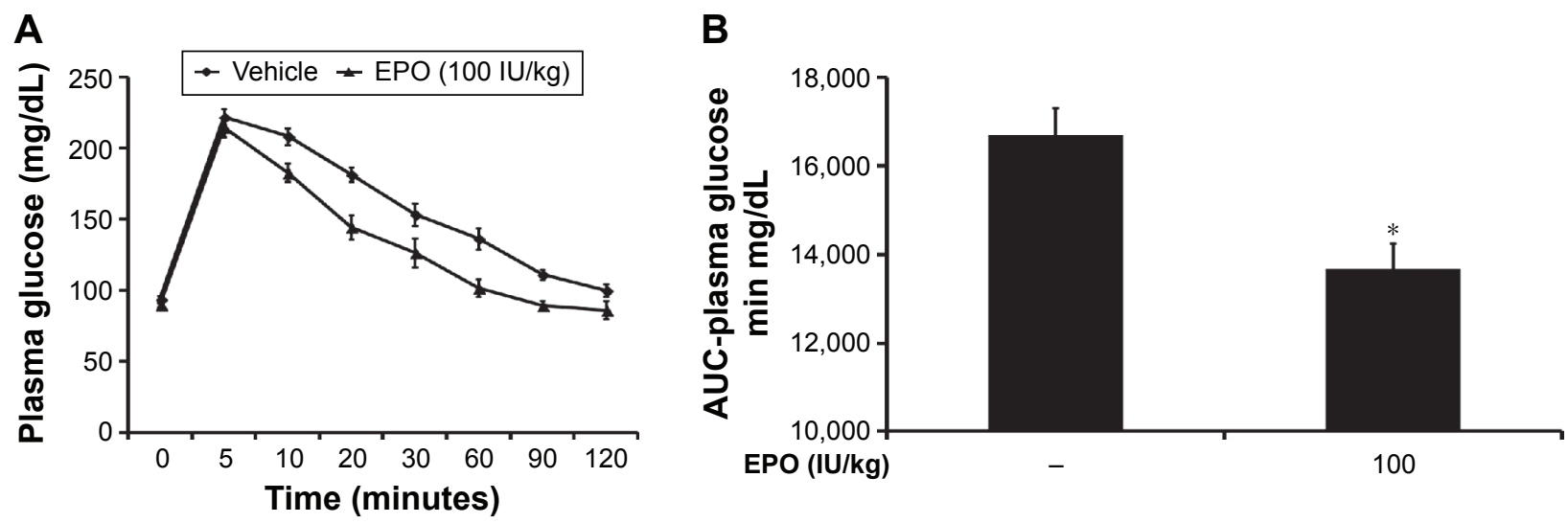

Figure 3 EPO-induced changes in blood glucose in rats measured using an IVGTT.

Notes: EPO-induced changes in blood glucose in normal rats that received an intravenous injection of glucose compared with that for same volume of vehicle are shown in (A). IVGTT was performed, and the calculated AUC values are compared in (B). Values (mean $\pm \mathrm{SE}$ ) were obtained from groups of eight animals. $* P<0.05$ compared with the vehicle-treated group.

Abbreviations: AUC, area under the curve; EPO, erythropoietin; IVGTT, intravenous glucose tolerance test; SE, standard error. 


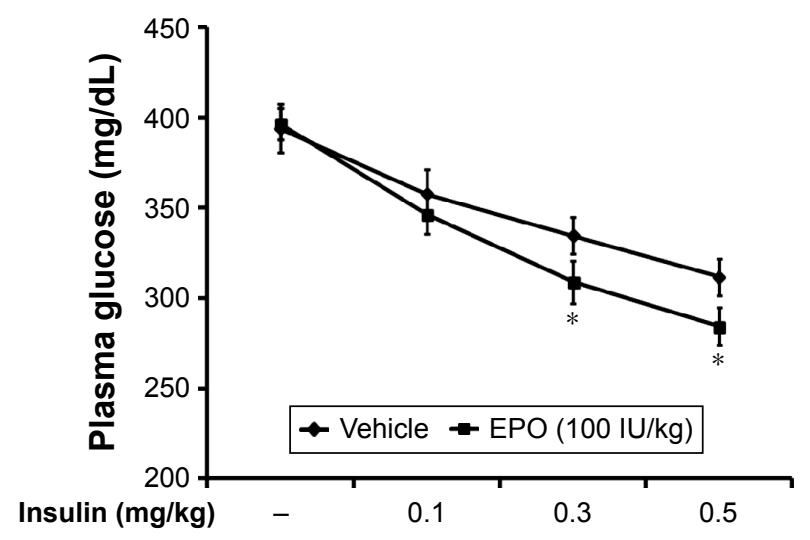

Figure 4 EPO-induced changes in insulin sensitivity in diabetic rats.

Notes: Blood glucose data from the diabetes model receiving vehicle treatment are shown as "Vehicle", and those from the EPO-treated diabetic rats are shown as "EPO" with the indicated dose. Values (mean $\pm \mathrm{SE}$ ) were obtained from groups of eight animals. $* P<0.05$ compared with the vehicle-treated diabetic rats.

Abbreviations: EPO, erythropoietin; SE, standard error.

of the STZ-diabetic rats. Repeated treatment with EPO resulted in an increase in p-AMPK (Figure 5B) similar to the GLUT4 changes described earlier. Therefore, the EPO-induced changes in p-AMPK were similar to the GLUT4 expression changes in the skeletal muscle of diabetic rats.

\section{Effect of EPO on the hepatic PEPCK changes in STZ-diabetic rats}

After the injection of EPO $(0.5 \mathrm{mg} / \mathrm{kg} / \mathrm{d})$ for 1 week, livers were also isolated from STZ-diabetic rats. In the Western blots, as shown in Figure 6A, the PEPCK protein level in the liver of STZ-diabetic rats was markedly increased to approximately twofold that in the normal rats. EPO produced a marked attenuation of the PEPCK protein level in diabetic rats (Figure 6A). This finding suggested that EPO improves diabetes via inhibition of hepatic PEPCK.

In addition, we also evaluated the changes in p-AMPK in liver tissues. As shown in Figure 6B, p-AMPK was significantly decreased in the livers of STZ-diabetic rats. After the same treatment as described earlier, EPO increased the levels of p-AMPK (Figure 6B). Therefore, it can be speculated that EPO increases $\mathrm{p}-\mathrm{AMPK}$ in liver, thus reversing the expression of PEPCK in diabetic rats.

\section{Discussion}

In the present study, we found that EPO attenuated hyperglycemia dose-dependently in type 1-like diabetic rats. In addition, blockade of the action of EPO using antibodies specific for EPORs indicated that this effect was mediated by EPOR activation. Thus, an insulinotropic action of EPO was identified in diabetic rats lacking insulin. Moreover, the insulin sensitivity was also enhanced by EPO. Therefore, similar to the suggestions in a recent review article, ${ }^{16} \mathrm{EPO}$ targeting for diabetes should offer the greatest promise for novel therapeutic strategies.

A bolus injection of EPO attenuated hyperglycemia in a dose-dependent manner, and this finding is consistent with the action of EPO in mice. ${ }^{17}$ In addition, hematopoietic parameters, including hematocrit and the number of red blood cells, were not modified by EPO treatment in diabetic rats. A nonhematopoietic action of EPO can thus be considered.
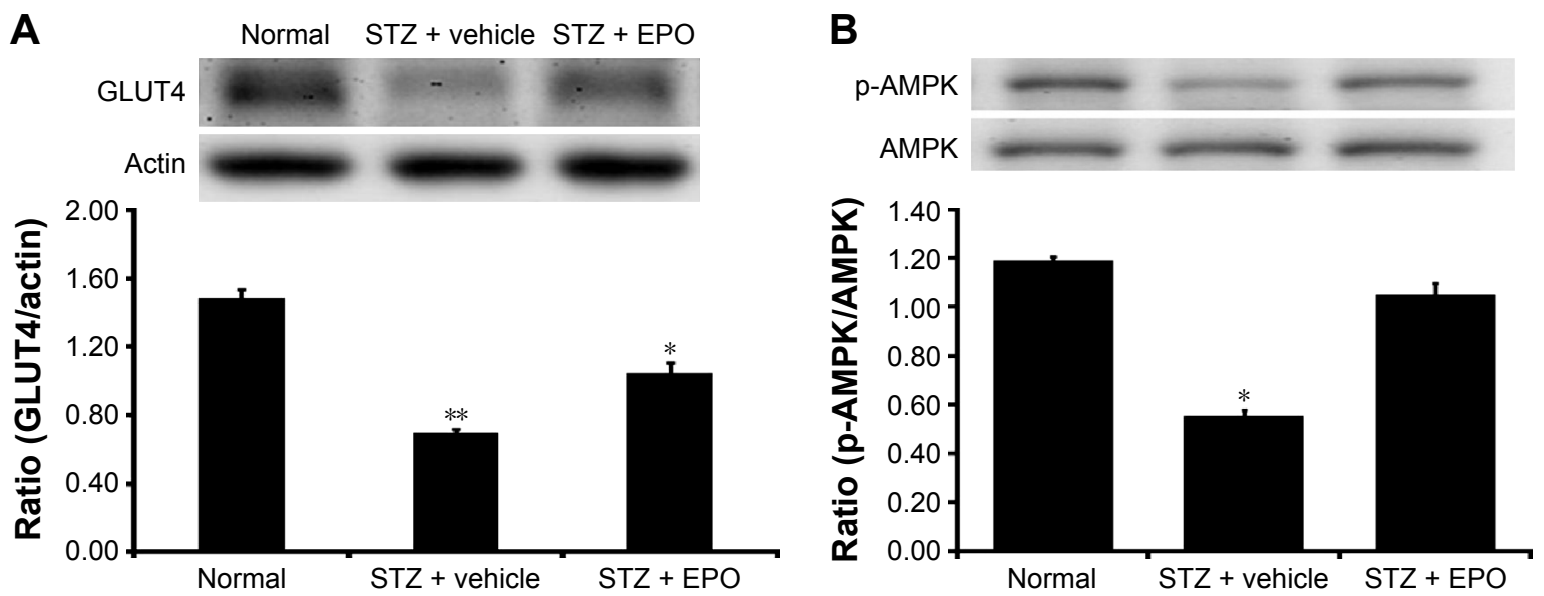

Figure 5 EPO-induced changes in GLUT4 expression (A) and AMPK phosphorylation (B) in the soleus muscle of type I-like diabetic rats.

Notes: Changes in GLUT4 expression (A) or phosphorylated AMPK (P-AMPK) (B) in rats with STZ-induced diabetes are shown as "STZ" for comparison with the changes in normal rats, "Normal". In addition, the EPO effects on diabetic rats are shown as "STZ + EPO" for comparison with diabetic rats that received vehicle only (STZ + Vehicle). Representative immunoblots are shown in the upper portion, and the relative expression levels of GLUT4/actin or P-AMPK/AMPK are shown in the lower column (mean $\pm \mathrm{SE}$ ). The data were obtained from six animals. $* P<0.05$ and $* * P<0.01$ compared with "Normal".

Abbreviations: AMPK, AMP-activated protein kinase; AMP, adenosine monophosphate; EPO, erythropoietin; GLUT4, glucose transporter 4; SE, standard error; STZ, streptozotocin. 
A

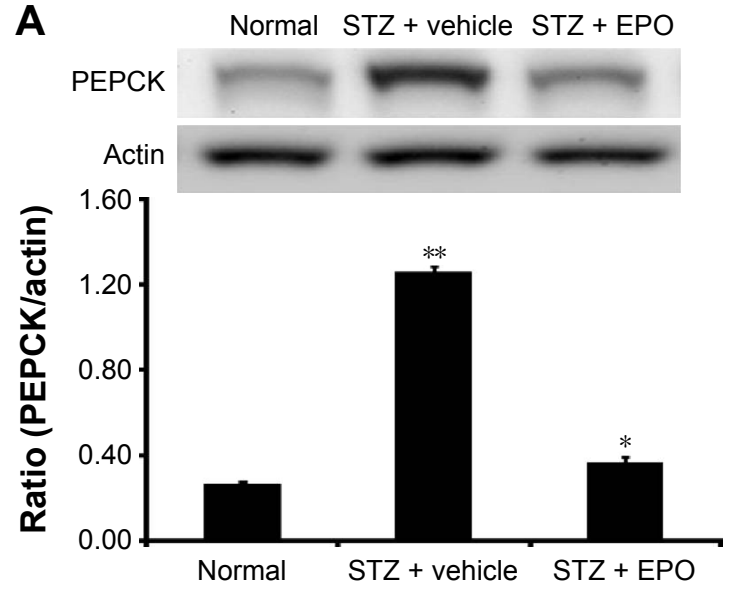

B

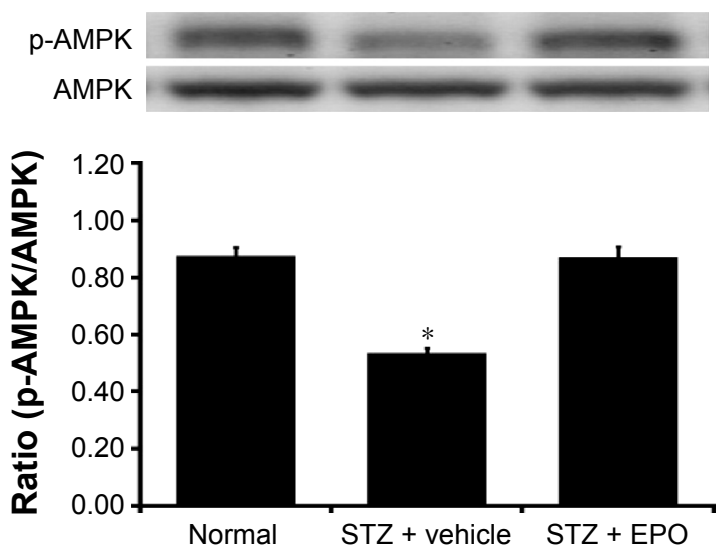

Figure 6 EPO-induced changes of PEPCK expression (A) and AMPK phosphorylation (B) in the liver of type I-like diabetic rats.

Notes: Changes in PEPCK expression (A) or P-AMPK (B) in rats with STZ-induced diabetes are shown as "STZ" for comparison with the changes in normal rats, "Normal". In addition, the effects of EPO on diabetic rats are shown as "STZ + EPO" with the indicated dose to compare the diabetic rats that received vehicle only (STZ + Vehicle). Representative immunoblots are shown in the upper portion, and the relative expression levels of PEPCK/actin or P-AMPK/AMPK are shown in the lower column (mean \pm SE). The data were obtained from six animals. $* P<0.05$ and $* * P<0.01$ compared with "Normal".

Abbreviations: AMPK, AMP-activated protein kinase; AMP, adenosine monophosphate; EPO, erythropoietin; P-AMPK, phosphorylated AMPK; PEPCK, phosphoenolpyruvate carboxykinase; SE, standard error; STZ, streptozotocin.

Furthermore, role of EPORs was investigated using the antibodies specific to EPOR as an alternative method, owing to the lack of a pharmacological antagonist. Basically, antibodies specific to receptor are usefully applied to identify the presence of receptors ${ }^{22}$ and to block the functions of receptor. ${ }^{23}$ Therefore, we employed antibodies specific to EPOR as the blocker in accordance with our previous report. ${ }^{24}$ As shown in Figure 2, the antibodies specific to EPOR inhibited the action of EPO, thus demonstrating that receptor activation mediates the EPO-induced hypoglycemia in diabetic rats lacking insulin. EPO-induced hypoglycemia has also been demonstrated in patients with type-1 diabetes. ${ }^{13}$ In addition, a positive effect of EPO on glucose homeostasis was observed in patients receiving hemodialysis. ${ }^{8}$ Therefore, EPO is useful in the reduction of hyperglycemia.

Regarding the possible mechanism(s) for EPO-induced attenuation of hyperglycemia, using the IVGTT, we found that EPO increases glucose utilization in a dose-dependent manner (Figure 3B). This result is consistent with a previous report regarding the EPO-induced improvement of glucose intolerance in diet-treated rats. ${ }^{15}$ Moreover, this pleiotropic action of EPO is not associated with endogenous insulin, indicating an insulinotropic action of EPO. Therefore, an increase of glucose utilization seems to be responsible for the reduction of hyperglycemia in the diabetic rats. Essentially, antioxidant-like substances are associated with the reduction of hyperglycemia in animals..$^{21,25} \mathrm{EPO}$ has been demonstrated to show antioxidant-like activity. ${ }^{16,26}$ Therefore, reduction of hyperglycemia induced by a bolus injection of EPO seems to be related to the antioxidant-like action, although more investigations are required to support this view. In addition, EPO enhanced the insulin sensitivity in diabetic rats. This result is reasonable because EPO has been linked to many signals that occur after activation of receptors. ${ }^{16}$ One of the marked signals associated with EPORs for glucose homeostasis is AMPK. ${ }^{16}$ In the present study, we also found that repeated EPO treatment might increase the phosphorylation of AMPK in muscles and livers of diabetic rats. In clinics, metformin is widely used to treat diabetes owing to activation of AMPK..$^{27,28}$ Therefore, it is clear that EPO is helpful in diabetes treatment.

A decrease in insulin-mediated glucose uptake caused by lower expression levels of GLUT4 has been documented in diabetic skeletal muscle, a major site for glucose disposal. ${ }^{29}$ Also, EPO is important in the regulation of glucose. ${ }^{30}$ In the present study, we found that repeated injection of EPO might reverse the decreased GLUT4 levels. This action is one of the potential mechanism(s) for the EPO-induced glucose uptake into muscles, which has been described previously in mice. ${ }^{31}$ Interestingly, EPO also increased the levels of p-AMPK in the muscle isolated from diabetic rats. It has been established that activation of AMPK may enhance GLUT4 levels. ${ }^{32}$ The current study is consistent with this view of the skeletal muscle in diabetic rats.

Hyperglycemia is a consequence of increased hepatic glucose output in concert with reduced peripheral glucose utilization in diabetes, and PEPCK is one of the key enzymes in the regulation of hepatic gluconeogenesis. ${ }^{33}$ Moreover, insulin deficiency is clearly associated with a change in hepatic metabolism. ${ }^{33}$ The production of EPO in the liver 
has been demonstrated. ${ }^{34}$ In the present study, we identified a marked increase in PEPCK expression in the liver isolated from type 1-like diabetic rats. Repeated treatment with EPO attenuated the higher expression of hepatic PEPCK. Previously, modification of PEPCK by EPO has not been demonstrated. Therefore, this study showed for the first time that EPO could influence hepatic gluconeogenesis through a reduction in PEPCK expression. In addition, an increase in AMPK phosphorylation seems to be associated with the reduction in PEPCK expression in the liver of diabetic rats that received EPO treatment. Counteracting changes in PEPCK and AMPK have been reported during cell aging. ${ }^{35}$ In addition, the natural product caffeamide also enhanced p-AMPK in liver to reduce the mRNA levels of PEPCK. ${ }^{36}$ However, the molecular mechanism(s) for this integration is still unknown and needs to be investigated in the future.

\section{Limitations}

Limitations of this study included the results being focused on the insulinotropic action of EPO, particularly the in vivo actions. Other actions of EPO, including the changes in blood lipids and endocrine functions, need be investigated in future. Moreover, cellular actions of EPO are also required to be clarified in the future.

Overall, injection of EPO is helpful for managing diabetic disorders, particularly hyperglycemia-associated changes. This finding is consistent with the suggestions from a recent review. ${ }^{37}$ Therefore, development of an EPO-like substance, either a peptide ${ }^{31,38}$ or a chemical compound, ${ }^{39}$ is useful for diabetes treatment in the future. The development of the anatagonist(s) and/or specific blockers to EPOR will assist the research in actions of EPO in advance. New classification of EPOR subtypes can thus be speculated. Therefore, new agent(s) related to EPOR will be developed for the applications in medical therapies for both hematopoietic and nonhematopoietic disorders.

\section{Conclusion}

Taken together, our results suggest that EPO is effective in reducing hyperglycemia in rats with type 1-like diabetes. Our findings highlight the pleiotropic action and/or the insulinotropic action of EPO associated with receptor activation. Therefore, in addition to development of agonist for EPO receptors, the application of EPO in diabetes treatment can be considered in clinics.

\section{Acknowledgments}

We thank Pei-Lin Chou and Yi-Zhi Chen for their assistance in our experiments. Thanks are also to American Journal Experts (AJE) for editing. The present study was supported in part by a grant from the Chi-Mei Medical Center in Taiwan, the Republic of China.

\section{Disclosure}

The authors report no conflicts of interest in this work.

\section{References}

1. Katz O, Barzilay E, Skaat A, Herman A, Mittelman M, Neumann D. Erythropoietin induced tumour mass reduction in murine lymphoproliferative models. Acta Haematol. 2005;114(3):177-179.

2. Eschbach JW, Kelly MR, Haley NR, Abels RI, Adamson JW. Treatment of the anemia of progressive renal failure with recombinant human erythropoietin. N Engl J Med. 1989;321(3):158-163.

3. Birgegard G, Aapro MS, Bokemeyer C, et al. Cancer-related anemia: pathogenesis, prevalence and treatment. Oncology. 2005;68(Suppl 1): $3-11$.

4. Maiese K, Chong ZZ, Shang YC, Wang S. Erythropoietin: new directions for the nervous system. Int J Mol Sci. 2012;13(9):11102-11129.

5. Ogilvie M, Yu X, Nicolas-Metral V, et al. Erythropoietin stimulates proliferation and interferes with differentiation of myoblasts. $J$ Biol Chem. 2000;275(50):39754-39761.

6. Nishimura K, Tokida M, Katsuyama H, Nakagawa H, Matsuo S. The effect of hemin-induced oxidative stress on erythropoietin production in HepG2 cells. Cell Biol Int. 2014;38(11):1321-1329.

7. Parsa CJ, Matsumoto A, Kim J, et al. A novel protective effect of erythropoietin in the infarcted heart. J Clin Invest. 2003;112(7): 999-1007.

8. Nairz M, Sonnweber T, Schroll A, Theurl I, Weiss G. The pleiotropic effects of erythropoietin in infection and inflammation. Microbes Infect. 2012;14(3):238-246.

9. Tuzcu A, Bahceci M, Yilmaz E, Bahceci S, Tuzcu S. The comparison of insulin sensitivity in non-diabetic hemodialysis patients treated with and without recombinant human erythropoietin. Horm Metab Res. 2004;36(10):716-720.

10. Schena FP, Gesualdo L. Pathogenetic mechanisms of diabetic nephropathy. J Am Soc Nephrol. 2005;16(Suppl 1):S30-S33.

11. Ohkubo Y, Kishikawa H, Araki E, et al. Intensive insulin therapy prevents the progression of diabetic microvascular complications in Japanese patients with non-insulin-dependent diabetes mellitus: a randomized prospective 6-year study. Diabetes Res Clin Pract. 1995; 28(2):103-117.

12. Shah SV, Baliga R, Rajapurkar M, Fonseca VA. Oxidants in chronic kidney disease. J Am Soc Nephrol. 2007;18(1):16-28.

13. Kristensen PL, Pedersen-Bjergaard U, Kjaer TW, et al. Influence of erythropoietin on cognitive performance during experimental hypoglycemia in patients with type 1 diabetes mellitus: a randomized cross-over trial. PLoS One. 2013;8(4):e59672.

14. Allegra V, Martimbianco L, Vasile A. Lipid and apolipoprotein patterns during erythropoietin therapy: roles of erythropoietin, route of administration, and diet. Nephrol Dial Transplant. 1997;12(5):924-932.

15. Caillaud C, Mechta M, Ainge H, et al. Chronic erythropoietin treatment improves diet-induced glucose intolerance in rats. J Endocrinol. 2015;225(2):77-88.

16. Maiese K. Erythropoietin and diabetes mellitus. World J Diabetes. 2015;6(14):1259-1273.

17. Katz O, Stuible M, Golishevski N, et al. Erythropoietin treatment leads to reduced blood glucose levels and body mass: insights from murine models. J Endocrinol. 2010;205(1):87-95.

18. Furman BL. Streptozotocin-induced diabetic models in mice and rats. Curr Protoc Pharmacol. 2015;70:5.47.1-20.

19. Cheng JT, Huang CC, Liu IM, Tzeng TF, Chang CJ. Novel mechanism for plasma glucose-lowering action of metformin in streptozotocininduced diabetic rats. Diabetes. 2006;55(3):819-825.

20. Sato K, Kitamura T, Kawamura G, et al. Glucose use in fasted rats under sevoflurane anesthesia and propofol anesthesia. Anesth Analg. 2013;117(3):627-633. 
21. Gulati V, Harding IH, Palombo EA. Enzyme inhibitory and antioxidant activities of traditional medicinal plants: potential application in the management of hyperglycemia. BMC Complement Altern Med. 2012;12:77.

22. Bord S, Ireland DC, Moffatt P, Thomas GP, Compston JE. Characterization of osteocrin expression in human bone. J Histochem Cytochem. 2005;53(10):1181-1187.

23. Ciardiello F, Tortora G. EGFR antagonists in cancer treatment. $N$ Engl J Med. 2008;358(11):1160-1174.

24. Li YX, Cheng KC, Asakawa A, et al. Role of musclin in the pathogenesis of hypertension in rat. PLoS One. 2013;8(8):e72004.

25. Shori AB. Screening of antidiabetic and antioxidant activities of medicinal plants. J Integr Med. 2015;13(5):297-305.

26. Rjiba-Touati K, Ayed-Boussema I, Guedri Y, Achour A, Bacha H, AbidEssefi S. Effect of recombinant human erythropoietin on mitomycin $\mathrm{C}$-induced oxidative stress and genotoxicity in rat kidney and heart tissues. Hum Exp Toxicol. 2016;35(1):53-62.

27. Cho K, Chung JY, Cho SK, et al. Antihyperglycemic mechanism of metformin occurs via the AMPK/LXRalpha/POMC pathway. Sci Rep. 2015;5:8145.

28. Gagnon J, Sheppard E, Anini Y. Metformin directly inhibits ghrelin secretion through AMP-activated protein kinase in rat primary gastric cells. Diabetes Obes Metab. 2013;15(3):276-279.

29. McGee SL, van Denderen BJ, Howlett KF, et al. AMP-activated protein kinase regulates GLUT4 transcription by phosphorylating histone deacetylase 5. Diabetes. 2008;57(4):860-867.

30. Montel-Hagen A, Sitbon M, Taylor N. Erythroid glucose transporters. Curr Opin Hematol. 2009;16(3):165-172.

31. Scully MS, Ort TA, James IE, et al. A novel EPO receptor agonist improves glucose tolerance via glucose uptake in skeletal muscle in a mouse model of diabetes. Exp Diabetes Res. 2011;2011:910159.
32. Rodriguez-Calvo R, Vazquez-Carrera M, Masana L, Neumann D. AICAR protects against high palmitate/high insulin-induced intramyocellular lipid accumulation and insulin resistance in HL-1 cardiac cells by inducing PPAR-target gene expression. PPAR Res. 2015; 2015:785783.

33. Consoli A, Nurjhan N, Capani F, Gerich J. Predominant role of gluconeogenesis in increased hepatic glucose production in NIDDM. Diabetes. 1989;38(5):550-557.

34. Koury ST, Bondurant MC, Koury MJ, Semenza GL. Localization of cells producing erythropoietin in murine liver by in situ hybridization. Blood. 1991;77(11):2497-2503.

35. Yuan Y, Hakimi P, Kao C, et al. Reciprocal changes in phosphoenolpyruvate carboxykinase and pyruvate kinase with age are a determinant of aging in caenorhabditis elegans. J Biol Chem. 2016; 291(3):1307-1319.

36. Kuo YH, Lin CH, Shih CC. Caffeamide 36-13 regulates the antidiabetic and hypolipidemic signs of high-fat-fed mice on glucose transporter 4, AMPK phosphorylation, and regulated hepatic glucose production. Evid Based Complement Alternat Med. 2014;2014:821569.

37. Wang L, Di L, Noguchi CT. Erythropoietin, a novel versatile player regulating energy metabolism beyond the erythroid system. Int J Biol Sci. 2014;10(8):921-939.

38. Heij L, Niesters M, Swartjes M, et al. Safety and efficacy of ARA 290 in sarcoidosis patients with symptoms of small fiber neuropathy: a randomized, double-blind pilot study. Mol Med. 2012;18:1430-1436.

39. Sanchis-Gomar F, Perez-Quilis C, Lippi G. Erythropoietin receptor (EpoR) agonism is used to treat a wide range of disease. Mol Med.2013; 19:62-64.
Drug Design, Development and Therapy

\section{Publish your work in this journal}

Drug Design, Development and Therapy is an international, peerreviewed open-access journal that spans the spectrum of drug design and development through to clinical applications. Clinical outcomes, patient safety, and programs for the development and effective, safe, and sustained use of medicines are a feature of the journal, which

\section{Dovepress}

has also been accepted for indexing on PubMed Central. The manuscript management system is completely online and includes a very quick and fair peer-review system, which is all easy to use. Visit http://www.dovepress.com/testimonials.php to read real quotes from published authors. 